unclassified Pulmonary Fibrosis, 67 (6.5\%) Fibrotic Hypersensitivity Pneumonitis, 100 (14\%) CT-ILD and 71 (9.9\%) exposure including Asbestos were classified. Crude prevalence rates (per 100’000 (Min-Max)) for Sarcoidosis 31.4 (11.1-67.5), UIP definite and probable 47.5 (12.2-76.9) and CT-ILD 23.1 (12.2-76.9). Prevalence of disease appeared to correlate with population density (figure 1) however there was a suggestion of clustering associated with topographic features particularly with regards to sarcoidosis.

Exploratory analysis of patient travel distance suggested that reallocation of 108 patients $(15 \%)$ to a satellite clinic closer to home could save in excess 14 '000 miles per year, reducing the carbon footprint by 4.15 tonnes.

Conclusions Northern Ireland has a stable population with little drift making it ideal for study. Preliminary analysis of data suggests intriguing clusters which may lead to insights into disease pathogenesis with further study. Analysis of crude prevalence and patient travel distance allows for planning of service provision and a potential reduction of carbon footprint.

\section{P3 PROGRESSION OF EARLY FIBROTIC ILA TO ESTABLISHED INTERSTITIAL LUNG DISEASE AND MORTALITY: OBSERVATIONS FROM A REGIONAL CENTRE}

${ }^{1}$ A Achaiah, ${ }^{2} \mathrm{P}$ Lyon, ${ }^{3} \mathrm{E}$ Fraser, ${ }^{3} \mathrm{P}$ Saunders, ${ }^{3} \mathrm{R}$ Hoyles, ${ }^{2} \mathrm{R}$ Benamore, ${ }^{1} \mathrm{LP}$ Ho. ${ }^{1} \mathrm{MRC}$ Human Immunology Unit, Weatherall Institute of Molecular Medicine, University of Oxford, Oxford, UK; ${ }^{2}$ Oxford Radiology Unit, Oxford University Hospitals NHS Foundation Trust, Oxford, UK; ${ }^{3}$ Oxford Interstitial Lung Disease Service, Oxford University Hospitals NHS Foundation Trust, Oxford, UK

\subsection{6/thorax-2021-BTSabstracts.113}

Introduction Interstitial lung abnormalities (ILA) are defined as patterns of increased lung density on CT scans, compatible with early interstitial lung disease (ILD). Patients are typically asymptomatic, but a proportion will progress to pulmonary fibrosis. Factors predisposing to this are unclear.

Aims Determine estimated prevalence of fibrotic ILAs in specified geographical region (Oxfordshire), and explore factors contributing to progression and mortality.

Methods Using the Clinical Record Interactive System database of the Oxford University Hospitals NHS trust (serving an estimated population of 800,000 ), we analysed reports from CTs performed between January-2015 and December-2020. Search criteria were selective for early fibrotic ILA within this database; ['reticulation' or 'interstitial'] AND ['sub-pleural' or 'basal' or 'lower zone' or 'Possible UIP'] AND [Age: 45-75]. Radiological features, patient demographics and contemporaneous blood leukocyte counts were examined to explore contribution to progression and mortality using multivariate Cox regression.

Results 40,711 patients underwent CT thorax during this period; 2735 (6.7\%) patients met inclusion criteria. Mean age 65.5 years $( \pm 7.32)$, male $1486(54.3 \%) .762$ of these patients had traction bronchiectasis and/or honeycombing on first CT. 355 cases had normal CTs; 80 had non-emphysematous cysts and GGO only in 279. The remaining 1259 cases demonstrated reticulation \pm additional parenchymal features (Table 1).

Follow on CT was performed in 390 cases. Progression was observed in 47.4\%; progression to reticulation $28(7.1 \%$ of $390)$, of reticulation $51(13.1 \%)$, to probable UIP $84(21.2 \%)$, to definite UIP $22(5.6 \%)$.

Compared to the group with normal CT, reticulation combined with emphysema \pm GGO demonstrated significant risk of mortality (Table1). Of those that underwent follow-on CT, progression appeared significantly associated with mortality [HR 1.92, 1.2-3.2, $\mathrm{p}=0.013$ ]. Progression to definite UIP was associated with greatest mortality risk [HR 3.47, 1.6-7.5, $\mathrm{p}=0.002]$. Monocyte:lymphocyte ratio $[1.50,1.16-1.94$, $p=0.002]$ and age $[1.04,1.01-1.07, p=0.010]$ at first CT scan, were the only independent factors associated with progression. Conclusion 3.1\% (1259/40,711) of patients requiring thoracic CT during our analysis period demonstrated ILA with early fibrotic features. Monocyte:lymphocyte ratio was significantly associated with progression suggesting this and other blood leukocyte derivations could be explored further as potential prognostic biomarkers of sub-clinical ILD.

\section{P4 $\quad$ LUNG CANCER SCREENING PROVIDES A UNIQUE OPPORTUNITY FOR EARLY DIAGNOSIS AND MANAGEMENT OF INTERSTITIAL LUNG DISEASES}

${ }^{1,2} \mathrm{RJ}$ Hewitt, ${ }^{3} \mathrm{EC}$ Bartlett, ${ }^{1} \mathrm{R}$ Ganatra, ${ }^{1} \mathrm{H}$ Butt, ${ }^{4} \mathrm{~J}$ Morjaria, ${ }^{1,2} \mathrm{~V}$ Kouranos, ${ }^{1,2} \mathrm{~F}$ Chua, ${ }^{1,2} \mathrm{M}$ Kokosi, ${ }^{1,2} \mathrm{PL}$ Molyneaux, ${ }^{2,3} \mathrm{SR}$ Desai, ${ }^{1,2} \mathrm{AU}$ Wells, ${ }^{1,2} \mathrm{RG}$ Jenkins, ${ }^{1,2} \mathrm{EA}$ Renzoni, ${ }^{4} \mathrm{~S}$ Kemp, ${ }^{2,3} \mathrm{~A}$ Devaraj, ${ }^{1,2} \mathrm{PM}$ George. ${ }^{1}$ Interstitial Lung Disease Unit, Royal Brompton and Harefield Hospitals, London, UK; ${ }^{2}$ The Margaret Turner Warwick Centre for Fibrosing Lung Diseases, NHLI, Imperial College London, London, UK; ${ }^{2}$ Department of Radiology, Royal Brompton and Harefield Hospitals, London, UK; ${ }^{4}$ Department of Respiratory Medicine, Royal Brompton \& Harefield Hospitals, London, UK

10.1136/thorax-2021-BTSabstracts. 114

Abstract P3 Table 1 Univariate and multivariate cox regression analysis for outcome of mortality. Hazard ratios of ILD categories are expressed relative to Nil ILD group

\begin{tabular}{|c|c|c|c|c|c|c|c|c|c|c|}
\hline \multicolumn{3}{|c|}{ Table 1. Outcome = Mortality } & \multicolumn{3}{|c|}{ Univariate } & \multicolumn{5}{|c|}{ Multivariate } \\
\hline Covariate & $\mathbf{n}$ & Death & HR & Lower & Upper & Sig. & HR & Lower & Upper & Sig. \\
\hline Age & - & - & 1.02 & 1.01 & 1.04 & $0.0001^{*}$ & 1.02 & 1.01 & 1.04 & $0.002^{*}$ \\
\hline Gender & - & - & 1.14 & 0.95 & 1.37 & 0.140 & 1.08 & 0.89 & 1.31 & 0.420 \\
\hline Nil ILD (ref. category) & $355(13.0 \%)$ & $43(12.1 \%)$ & - & - & - & $<0.001^{*}$ & - & - & - & $<0.001^{*}$ \\
\hline GGO only & $279(10.2 \%)$ & $54(19.3 \%)$ & 1.67 & 1.12 & 2.50 & $0.011^{*}$ & 1.63 & 1.09 & 2.43 & $0.017^{*}$ \\
\hline Reticulation only & $603(22.0 \%)$ & $68(11.3 \%)$ & 1.09 & 0.74 & 1.59 & 0.667 & 1.01 & 0.69 & 1.48 & 0.966 \\
\hline Reticulation + GGO & $373(13.6 \%)$ & $55(14.7 \%)$ & 1.43 & 0.96 & 2.13 & 0.079 & 1.39 & 0.93 & 2.07 & 0.108 \\
\hline Reticulation + Emph & $133(4.9 \%)$ & $28(2.1 \%)$ & 2.32 & 1.45 & 3.71 & $<0.001^{*}$ & 2.08 & 1.29 & 3.35 & $0.003^{*}$ \\
\hline Retic + GGO + Emph & $150(5.5 \%)$ & $27(18.0 \%)$ & 1.74 & 1.07 & 2.81 & $0.025^{*}$ & 1.67 & 1.03 & 2.70 & $0.038^{*}$ \\
\hline Probable UIP & $490(17.9 \%)$ & $86(17.5 \%)$ & 1.58 & 1.10 & 2.28 & $0.014^{*}$ & 1.45 & 1.01 & 2.11 & $0.047^{*}$ \\
\hline Definite UIP & $272(9.9 \%)$ & $87(32.0 \%)$ & 2.82 & 1.95 & 4.10 & $<0.001^{*}$ & 2.55 & 1.76 & 3.69 & $<0.001^{*}$ \\
\hline
\end{tabular}

* P<0.05. GGO; ground glass opacities, UIP; Usual interstitial pneumonia, Emph; Emphysema 